\title{
EXISTENCE OF THE FOCK REPRESENTATION FOR CURRENT ALGEBRAS OF THE GALILEI ALGEBRA
}

\author{
LUIGI ACCARDI, ANDREAS BOUKAS, AND JOLANTA MISIEWICZ
}

\section{INTRODUCTION AND STATEMENT OF THE PROBLEM}

The investigation of the 3 problems stated below has led, in the past 10 years, to a multiplicity of new results and to the discovery of several unexpected connections between different fields of mathematics and physics:

Problem I: construct a continuous analogue of the $*$-Lie algebra (and associative $*$-algebra) of differential operators in $d$ variables with polynomial coefficients

$$
D O P C\left(\mathbb{R}^{d}\right):=\left\{\sum_{n \in \mathbb{N}^{d}} P_{n}(x) \partial_{x}^{n} ; x \in \mathbb{R}^{d} ; P_{n} \text { complex polynomials in } d \text { real variables }\right\}
$$

where continuous means that the space

$$
\mathbb{R}^{d} \equiv\{\text { functions }\{1, \ldots, d\} \rightarrow \mathbb{R}\}
$$

is replaced by some function space

$$
\{\text { functions } \mathbb{R} \rightarrow \mathbb{R}\}
$$

Since, for $d=1$, this algebra can be canonically identified to the universal enveloping algebra of the onemode Heisenberg algebra $\mathrm{Heis}_{\mathbb{C}}(1)$, this problem is equivalent to the old standing problem of constructing a theory of nonlinear quantum (boson) fields: hence its connections with the renormalization problem.

Problem II: construct $*-$ representations of this algebra (typically a generalization of the Fock representation) as operators acting on some domain in a Hilbert space $\mathcal{H}$

Problem III: prove the unitarity of these representations, i.e. that the skew symmetric elements of this *-algebra can be exponentiated, leading to strongly continuous 1-parameter unitary groups.

The combination of the constructive results obtained in this direction with the no-go theorems have made clear since the early developments of this programme, that the algebra $D O P C\left(\mathbb{R}^{d}\right)$ is too large to allow a nontrivial realization of this programme and that one has to restrict one's attention to appropriately chosen subalgebras of it (see the survey paper [7]).

The investigation of the connections of the renormalization problem with the problem of central extensions of $*$-Lie algebras has led to the discovery that the one--mode Heisenberg algebra $H e i s_{\mathbb{C}}(1)$ admits a unique non trivial central extension and to its boson representation.

More precisely: let $a, a^{\dagger}, h$ (central element) denote the generators of the one-mode Heisenberg algebra Heis $_{\mathbb{C}}(1)$, with relations

$$
\begin{gathered}
(a)^{*}=a^{\dagger} ; h^{*}=h \\
{\left[a, a^{\dagger}\right]_{\text {Heis }}=h \quad ; \quad[a, h]_{\text {Heis }}=\left[h, a^{\dagger}\right]_{\text {Heis }}=0}
\end{gathered}
$$

Date: July 14, 2010.

Key words and phrases. Galilei algebra, Heisenberg algebra, Central extension, Fock representation, Positive definite kernel, Infinitely divisible distribution. 
Here and in what follows all omitted commutators are assumed to be equal to zero. We define $p$ and $q$ by the equations

$$
a^{\dagger}=p+i q \quad ; \quad a=p-i q
$$

and we suppose that $a^{\dagger}, a, h, p, q$ are realized in the Schroedinger representation, where the powers of all these operators are well defined. The following result was proved in [6] and [2], see also [8] and [9].

Theorem 1. Up to isomorphisms there exists a unique nontrivial central extension of the Heisenberg algebra Heis $_{\mathbb{C}}(1)$, denoted $C e H e i s_{\mathbb{C}}(1)$, with generators $a, a^{\dagger}, h, E$ (central element) and relations

$$
\begin{array}{rlrl}
{\left[a, a^{\dagger}\right]_{\text {CeHeis }}} & =h \quad ; \quad\left[h, a^{\dagger}\right]_{\text {CeHeis }}=E & ; & {[a, h]_{\text {CeHeis }}=E} \\
(a)^{*}=a^{\dagger} & , \quad h^{*}=h \quad, & E^{*}=E
\end{array}
$$

Furthermore (boson representation of $\left.\mathrm{CeHeis}_{\mathbb{C}}(1)\right)$ :

(i) one can choose constants $A, B, C, D \in \mathbb{C}$ such that the elements

$$
\begin{aligned}
p_{C H}^{2} & :=\frac{B a^{\dagger}-\bar{B} a}{A \bar{B}-\bar{A} B} \\
q_{C H} & :=\frac{A a^{\dagger}-\bar{A} a}{A \bar{B}-\bar{A} B} \\
p_{C H} & :=h / 2 i D
\end{aligned}
$$

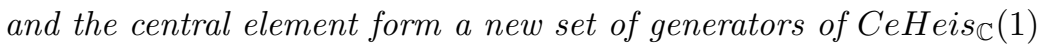

(ii) the map

$$
\left(p_{C H}^{2}, p_{C H}, q_{C H}, E\right) \mapsto\left(p^{2}, p, q, 1\right) \equiv\left(q^{2}, q, p, 1\right)
$$

extends to $a *$-Lie algebra isomorphism between $\mathrm{CeHeis}_{\mathbb{C}}(1)$ and the Galilei algebra $\left(q^{2}, q, p, 1\right)$.

Various realizations of the Galilei algebra were well known and studied both in the Mathematical and Physics literature (Bourbaki [12], Feinsilver and Schott [14], Franz [16], Ovando [18], ...). The apparently new point in the above theorem was the identification of this algebra with the unique nontrivial central extension of the Heisenberg algebra.

The continuous extension of $\mathrm{CeHeis}_{\mathbb{C}}(1)$, i.e. the current algebra of this $*$-Lie algebra over $\mathbb{R}$ (all what we say in the following remains valid for $\mathbb{R}^{d}$ for any $d \in \mathbb{N}$ ), has generators (in the sense of operator valued distributions, see [11])

$$
\left\{q_{s}^{2}, q_{s}, p_{t}: s, t \in \mathbb{R}\right\}
$$

with brackets and involution well defined and deduced from the corresponding brackets and involution of the usual (linear) free boson field in momentum representation:

$$
\begin{gathered}
{\left[a_{t}, a_{s}^{\dagger}\right]=\delta(t-s) ;\left[a_{t}^{\dagger}, a_{s}^{\dagger}\right]=\left[a_{t}, a_{s}\right]=0} \\
\left(a_{s}\right)^{*}=a_{s}^{\dagger}
\end{gathered}
$$

Therefore, for the Galilei algebra, Problem (I) above is easily solved.

The solution of Problem (II) for this algebra, namely:

Can one construct a generalization of the Fock $*$-representations for the current algebra of CeHeis $\mathbb{C}(1)$ over $\mathbb{R}$ ?

was open and, by a result of Accardi and Boukas obtained in [2], was reduced to the proof of the infinite divisibility of the vacuum distribution of the self-adjoint elements of $\mathrm{CeHeis}_{\mathbb{C}}(1)$.

This proof was recently obtained in a joint paper (cf. [10]) by L. Accardi, A. Boukas and J. Misiewicz and in the following we outline the main steps of the argument. 
The first step is to compute the vacuum characteristic function of the self-adjoint elements of $\mathrm{CeHeis} \mathbb{C}(1)$ (see section 2 below). Then we split this characteristic function as a product of two factors, one of which is the characteristic function of the Gamma distribution and, by a scaling argument we reduce the proof of infinite divisibility to the proof that the remaining factor is also the characteristic function of some probability distribution i.e., by Bochner's theorem, that it is positive definite and continuous at 0. This last statement is proved by adapting to our goals the Fock space characterization of infinitely divisible measures on Lie groups as developed by Araki, Woods, Parthasarathy, Schmidt, Guichardet (see the monographs [19], [17] and the bibliography therein).

In the Appendix of the present paper we give a synthetic re-formulation, with a few integrations, of the part of the theory that is needed to deduce the above mentioned theorem.

\section{RANDOM VARIABLES In $\mathrm{CeHeis}_{\mathbb{C}}(1)$}

As explained in section (1) we suppose that $p, q$ are realized in the one mode Schroedinger representation where we know that the operators of the form

$$
X:=L p^{2}+B p+C q \quad ; \quad L, B, C \in \mathbb{R}
$$

are essentially self-adjoint, so that their vacuum characteristic functions

$$
\left\langle\Phi, e^{i s X} \Phi\right\rangle \quad ; \quad s \in \mathbb{R}
$$

are well defined. In terms of $\left\{b^{+2}, b^{2}, b^{+} b, b^{+}, b, 1\right\}$, with the replacements

$$
-L / 4 \rightarrow L \quad ; \quad B / 2, C / 2 \rightarrow B, C
$$

and with the notation

$$
M:=C+i B
$$

we obtain

$$
X=L b^{+2}+L b^{2}-2 L b^{+} b+\bar{M} b^{\dagger}+M b-L
$$

Our first goal is to compute the characteristic function of $X$. To achieve this goal we use the following splitting formula proved in [2].

Lemma 1. (Splitting formula) Let $L \in \mathbb{R}$ and $M, N \in \mathbb{C}$. Then for all $s \in \mathbb{R}$ such that 2 L $s+1>0$

$$
e^{s\left(L b^{2}+L b^{\dagger 2}-2 L b^{\dagger} b-L+M b+N b^{\dagger}\right)} \Phi=e^{w_{1}(s) b^{\dagger 2}} e^{w_{2}(s) b^{\dagger}} e^{w_{3}(s)} \Phi
$$

where

$$
\begin{gathered}
w_{1}(s)=\frac{L s}{2 L s+1} \\
w_{2}(s)=\frac{L(M+N) s^{2}+N s}{2 L s+1} \\
w_{3}(s)=\frac{(M+N)^{2}\left(L^{2} s^{4}+2 L s^{3}\right)+3 M N s^{2}}{6(2 L s+1)}-\frac{\ln (2 L s+1)}{2}
\end{gathered}
$$

Putting $N:=\bar{M}$ in (2.4) we obtain

$$
e^{s X} \Phi=e^{s\left(-L b^{2}-L b^{\dagger 2}+2 L b^{\dagger} b+L+M b+\bar{M} b^{\dagger}\right)} \Phi=e^{w_{1}(s) b^{\dagger 2}} e^{w_{2}(s) b^{\dagger}} e^{w_{3}(s)} \Phi
$$

where

$$
\begin{aligned}
w_{1}(s) & =\frac{L s}{2 L s+1} \\
w_{2}(s) & =\frac{2 R e(M) L s^{2}+\bar{M} s}{2 L s+1}
\end{aligned}
$$




$$
w_{3}(s)=\frac{4 B^{2}\left(L^{2} s^{4}+2 L s^{3}\right)+3|M|^{2} s^{2}}{6(2 L s+1)}-\frac{\ln (2 L s+1)}{2}
$$

By analytic continuation (in $s$ )

$$
e^{w_{3}(i s)}=(1+2 L i s)^{-1 / 2} e^{\frac{4 B^{2}\left(L^{2} s^{4}-2 i L s^{3}\right)-3|M|^{2} s^{2}}{6(1+i 2 L s)}}
$$

In conclusion: the characteristic function of the vacuum distribution of the operator random variable

$$
X=L q^{2}+B q+C p
$$

is

$$
\begin{gathered}
\left\langle\Phi, e^{i s\left(L q^{2}+B q+C p\right)} \Phi\right\rangle=(1-2 L i s)^{-1 / 2} e^{\frac{4 B^{2}\left(L^{2} s^{4}+2 i L s^{3}\right)-3|M|^{2} s^{2}}{6(1-i 2 L s)}} \\
=:(1-2 L i s)^{-1 / 2} e^{\psi_{L}(s, M)}
\end{gathered}
$$

\section{INFINITE DIVISIBILITY}

Recall, from equation (2.10), the definition

$$
\psi_{L}(s, M):=\frac{4 \operatorname{Re}(M)^{2} L s^{3}(L s+2 i)-3|M|^{2} s^{2}}{6(1-2 i L s)}
$$

and notice two important remarks

(i) (scaling property) for every $t \in \mathbb{R}_{+}$

$$
\psi_{L}(s, \sqrt{t} M):=t \frac{4 R e(M)^{2} L s^{3}(L s+2 i)-3|M|^{2} s^{2}}{6(1-2 i L s)}=t \psi_{L}(s, M)
$$

(ii) for every $L \in \mathbb{R}$ and $M \in \mathbb{C}$,

$$
\varphi_{L, M}(s):=(1-2 L i s)^{-1 / 2} e^{\psi_{L}(s, M)}
$$

where $\varphi_{L}(s, M)$, given by the left hand side of $(2.10)$, is a characteristic function for every $t \in \mathbb{R}_{+}$and for every $L \in \mathbb{R}$ and $M \in \mathbb{C}$.

Denoting

we have that

$$
\varphi(s):=(1-2 L i s)^{-1 / 2}
$$

$$
\varphi_{L, M}(s):=\varphi(s) e^{\psi_{L}(s, M)}
$$

Suppose that one can prove that, for every $L \in \mathbb{R}$ and $M \in \mathbb{C}$, the function

$$
e^{\psi_{L}(s, M)}
$$

is a characteristic function. Then this characteristic function is infinitely divisible because the scaling property implies that, for any $t \geq 0$ :

$$
\left(e^{\psi_{L}(s, M)}\right)^{t}=e^{t \psi_{L}(s, M)}=e^{\psi_{L}(s, \sqrt{t} M)}
$$

which is a characteristic function because of our assumption.

Therefore the left hand side of (3.3), which is already known to be a characteristic function, is infinitely divisible being the product of two infinitely divisible characteristic functions.

The proof that, for every $L \in \mathbb{R}$ and $M \in \mathbb{C}$, the function $e^{\psi_{L}(s, M)}$ is a characteristic function depends on the following result.

Theorem 2. In the semigroup, for pointwise multiplication, of positive definite kernels on $\mathbb{R}$ (the choice of $\mathbb{R}$ is irrelevant), the infinitely divisible positive definite kernels form a subgroup. 
The proof of this theorem follows from the general theory of infinitely divisible positive definite kernels as developed in the monograph [19].

\subsection{A particular case.}

\section{Theorem 3. If}

$$
B=\operatorname{Re}(M)=0 \Leftrightarrow M \in i \mathbb{C}
$$

the vacuum characteristic functions given by (2.10) are infinitely divisible with cumulant generating function given by

$$
-i \frac{|M|^{2}}{4 L} s-\int_{0}^{\infty}\left(1-e^{i s u}\right) \frac{1 / 2}{u} e^{-2 L u} d u-\frac{|M|^{2}}{8 L^{2}} \int_{0}^{\infty}\left(1-e^{i s u}\right) e^{-2 L u} d u
$$

Remark: Formula (3.5) gives also the explicit form of the associated Levy measure.

\section{APPENDIX: INFINITELY DIVISIBLE KERNELS}

\section{KERNELS AND MATRiCES}

Definition 1. Let $S$ be a set, a function $K:(x, y) \in S \times S \rightarrow k(x, y) \in \mathbb{C}$ is called a $\mathbb{C}$-valued kernel on $S$ (only kernel if no ambiguity is possible). If $S$ is a finite set, then there exists a $d \in \mathbb{N}$ such that, up to relabeling the elements of $S$ can be identified to the subset $\{1, \ldots, d\} \subseteq \mathbb{N}$. With this identification a kernel on $S$ is identified to the $d \times d$ complex matrix

$$
k(i, j)=: k_{i, j} \quad ; \quad i, j \in\{1, \ldots, d\}
$$

4.1. Positive definite matrices. In the following $\mathbb{C}$ will be considered as a Hilbert space with scalar product $\langle x, y\rangle:=\bar{x} y$ so that $\mathcal{B}(\mathbb{C})$ is identified to $\mathbb{C}$ acting on itself by multiplication. For $d \in \mathbb{N}$ and $k=\left(k_{i j}\right) \in M_{d}(\mathbb{C})$ and $x_{1}, \cdots, x_{d} \in \mathbb{C}$ we will use the notation

$$
\bar{x}_{i} k_{i j} x_{j}:=\sum_{i, j=1}^{d} \bar{x}_{i} k_{i j} x_{j}=\langle x, k y\rangle
$$

(i.e. we assume summation over repeated indices) where $\langle\cdot, \cdot\rangle$ denotes the hermitian scalar product on $\mathbb{C}^{d}$

$$
\langle x, y\rangle:=\sum_{j=1}^{d} \bar{x}_{j} y_{j} \quad ; \quad x=\left(x_{1}, \cdots, x_{d}\right), y=\left(y_{1}, \cdots, y_{d}\right) \in \mathbb{C}^{d}
$$

$\mathbb{R}^{d}$ is identified to the subspace of $\mathbb{C}^{d}$ obtained as range of the projection

$$
x=\left(x_{1}, \cdots, x_{d}\right) \in \mathbb{C}^{d} \mapsto \operatorname{Re}(x):=\left(\operatorname{Re}\left(x_{1}\right), \cdots, \operatorname{Re}\left(x_{d}\right)\right)
$$

The restriction on $\mathbb{R}^{d}$ of the hermitian scalar product on $\mathbb{C}^{d}$ is real valued. The $d \times d$ complex matrices $M_{d}(\mathbb{C})$ act naturally on $\mathbb{C}^{d}$; this action induces a action of the $d \times d$ real matrices $M_{d}(\mathbb{R})$ on $\mathbb{C}^{d}$.

The adjoint $k^{*}$ of a matrix $k=\left(k_{i j}\right)$ is defined by

$$
\langle k x, y\rangle=\left\langle x, k^{*} y\right\rangle \quad ; \quad x=\left(x_{1}, \cdots, x_{d}\right), y=\left(y_{1}, \cdots, y_{d}\right) \in \mathbb{C}^{d}
$$

Thus

$$
\left(k^{*}\right)_{i j}=\overline{k_{j i}}
$$

Definition 2. A matrix $k=\left(k_{i j}\right) \in M_{d}(\mathbb{C})$ is called:

(i) Hermitian if

(ii) skew-Hermitian if

$$
\langle x, k x\rangle=\bar{x}_{i} k_{i j} x_{j} \in \mathbb{R} \quad ; \quad \forall x_{1}, \cdots, x_{d} \in \mathbb{C}
$$

$$
\langle x, k x\rangle=\bar{x}_{i} k_{i j} x_{j}=0 \quad ; \quad \forall x_{1}, \cdots, x_{d} \in \mathbb{C}
$$


(iii) symmetric if it is Hermitian and with real coefficients, i.e.

$$
k=\left(k_{i j}\right) \in M_{d}(\mathbb{R})
$$

(iv) symplectic if it is skew-Hermitian and with real coefficients, i.e.

$$
k=\left(k_{i j}\right) \in M_{d}(\mathbb{R}) \quad ; \quad\langle x, k x\rangle=\bar{x}_{i} k_{i j} x_{j}=0 \quad ; \quad \forall x_{1}, \cdots, x_{d} \in \mathbb{C}
$$

Lemma 2. A matrix $k=\left(k_{i j}\right) \in M_{d}(\mathbb{C})$ is:

(i) Hermitian if and only if

$$
k_{j i}=\overline{k_{i j}} \Leftrightarrow k=k^{*} \quad ; \quad \forall x_{1}, \cdots, x_{d} \in \mathbb{C}
$$

In particular $k=\left(k_{i j}\right) \in M_{d}(\mathbb{C})$ is Hermitian if and only if its restriction on $\mathbb{R}^{d}$ is Hermitian.

(ii) skew-Hermitian if and only if its diagonal coefficients are zero and

$$
k_{j i}=-\overline{k_{i j}} \Leftrightarrow k=-k^{*} \quad ; \quad \forall x_{1}, \cdots, x_{d} \in \mathbb{C} ; i \neq j
$$

(iii) symplectic if and only if $\forall i, j \in\{1, \cdots, d\}$

$$
k_{i j}=-k_{j i}
$$

(iv) skew-Hermitian and Hermitian if and only if its diagonal coefficients are zero and its off-diagonal coefficients are purely imaginary.

Proof. Let $k=\left(k_{i j}\right) \in M_{d}(\mathbb{C}) . k$ is Hermitian if and only if $\forall x_{1}, \cdots, x_{d} \in \mathbb{C}$

$$
\langle x, k x\rangle=\bar{x}_{i} k_{i j} x_{j}=\left(\bar{x}_{i} k_{i j} x_{j}\right)^{-}=x_{i} \bar{k}_{i j} \bar{x}_{j}=\bar{x}_{i} \bar{k}_{j i} x_{j}
$$

Since $x_{1}, \cdots, x_{d} \in \mathbb{C}$ are arbitrary, this is equivalent to (4.1). The restriction to $\mathbb{R}^{d}$ of the above identity gives

From this (4.1) follows by choosing $\forall i_{0}, j_{0} \in\{1, \cdots, d\}$

$$
x_{i} k_{i j} x_{j}=x_{i} \bar{k}_{j i} x_{j}
$$

$$
x_{j}=\delta_{i_{0, i}} \quad ; \quad y_{i}=\delta_{j_{0, j}}
$$

$k$ is skew-Hermitian if and only if $\forall x_{1}, \cdots, x_{d} \in \mathbb{C}$

$$
\begin{gathered}
0=\langle x+y, k(x+y)\rangle=\langle x, k y\rangle+\langle y, k x\rangle \Leftrightarrow\langle x, k y\rangle=-\langle y, k x\rangle \\
\Leftrightarrow \bar{x}_{i} k_{i j} y_{j}=-\bar{y}_{j} k_{j i} x_{i}
\end{gathered}
$$

Fixing $i_{0}, j_{0} \in\{1, \cdots, d\}$ and choosing

$$
x_{j}=\delta_{i_{0, i}} \quad ; \quad y_{i}=\delta_{j_{0, j}}
$$

one finds

$$
k_{i_{0} j_{0}}=-k_{j_{0} i_{0}}
$$

Finally $k=\left(k_{i j}\right) \in M_{d}(\mathbb{C})$ is skew-Hermitian and Hermitian if and only if

i.e. $k_{i i}=0$ and $k_{j i}$ is purely imaginary for $i \neq j$.

$$
k_{j i}=-k_{i j}=-\bar{k}_{j i} \quad ; \quad \forall i, j \in\{1, \cdots, d\}
$$

Lemma 3. A matrix $h=\left(h_{i j}\right) \in M_{d}(\mathbb{C})$ is Hermitian if and only if the matrix $\operatorname{Re}(h):=\left(\operatorname{Re}\left(h_{i j}\right)\right) \in M_{d}(\mathbb{R})$ is symmetric, i.e.

$$
R e\left(h_{i j}\right)=R e\left(h_{j i}\right)
$$

and the matrix $\operatorname{Im}(h):=\left(\operatorname{Im}\left(h_{i j}\right)\right) \in M_{d}(\mathbb{R})$ is symplectic, i.e.

$$
\operatorname{Im}\left(h_{i j}\right)=-\operatorname{Im}\left(h_{j i}\right)
$$


Proof. $h=\left(h_{i j}\right)$ is Hermitian if and only if

$$
\operatorname{Re}\left(h_{i j}\right)-i \operatorname{Im}\left(h_{i j}\right)=\bar{h}_{i j}=h_{j i}=\operatorname{Re}\left(h_{j i}\right)+i \operatorname{Im}\left(h_{j i}\right)
$$

and this is equivalent to the identities (4.3) and (4.4).

Definition 3. A matrix $\left(k_{i j}\right) \in M_{d}(\mathbb{C})$ is said to be positive definite if

$$
\sum_{i, j=1}^{d} x_{i}^{*} k_{i j} x_{j} \geq 0
$$

$\forall x_{1}, \ldots, x_{d} \in \mathbb{C}$. If in addition

$$
\sum_{i, j=1}^{d} x_{i}^{*} k_{i j} x_{j}=0 \Leftrightarrow x_{1}=\cdots=x_{d}=0
$$

then the matrix $\left(k_{i j}\right)(i, j=1, \ldots, d)$ is called strictly positive definite.

Remark: Note that the diagonal elements of a positive definite matrix are positive because $\forall x \in \mathbb{C}$

$$
x^{*} k_{i i} x=|x|^{2} k_{i i} \geq 0
$$

Lemma 4. $k \in M_{d}(\mathbb{C})$ is $P D$ if and only if for every skew-Hermitian matrix $S \in M_{d}(\mathbb{C}), k+S$ is $P D$

Proof. Clear from the definition of PD.

Remark: If $k$ is positive definite and Hermitian and $S \in M_{d}(\mathbb{C})$ is symplectic, then $k+S$ is PD but it is Hermitian if and only if $S$ is. If this is the case, being also skew-Hermitian, $S$ must be symplectic by Lemma (2) hence its diagonal coefficients are zero and its off-diagonal coefficients are purely imaginary. Thus there exist matrices which are positive definite but not Hermitian.

Lemma 5. A matrix $k \in M_{d}(\mathbb{C})$ is positive definite and Hermitian if and only if there exist two Hermitian matrices a, $b \in \mathrm{Herm}_{d}(\mathbb{C})$ such that

$$
k=\left(a^{2}+b^{2}\right)+i(a b-b a)
$$

Remark: In particular any such $k$ has the form

$$
k=k_{H,+}+i k_{S}
$$

with $k_{H,+} \mathrm{PDH}$ and $k_{S}$ skew adjoint. This decomposition is highly non unique, reflecting the no uniqueness of the square root.

Proof. $k$ is positive definite and Hermitian if and only if there exists a matrix $x \in M_{d}(\mathbb{C})$ such that $k=x^{*} x$. Writing

$$
x=\frac{1}{2}\left(x+x^{*}\right)+\frac{1}{2}\left(x-x^{*}\right)=: a+i b
$$

by construction $a$ and $b$ are Hermitian. Therefore $k$ is positive definite if and only if it has the form

$$
k=(a+i b)^{*}(a+i b)=(a-i b)(a+i b)=\left(a^{2}+b^{2}\right)+i(a b-b a)
$$


Remark: Using the identity (4.9) any matrix $k$ can be written in the form

$$
k=k_{H}+i k_{S}=\operatorname{Re}\left(k_{H}\right)+i \operatorname{Im}\left(k_{H}\right)+i \operatorname{Re}\left(k_{S}\right)-\operatorname{Im}\left(k_{S}\right)
$$

where

$$
k_{H}=k_{H}^{*} \quad ; \quad k_{S}=k_{S}^{*}
$$

$\operatorname{Re}\left(k_{H}\right), \operatorname{Re}\left(k_{S}\right)$ are Hermitian and $\operatorname{Im}\left(k_{H}\right), \operatorname{Im}\left(k_{S}\right)$ are skew-symmetric.

Lemma 6. $k=\left(k_{i j}\right)$ is positive definite if and only if, in the notation (4.10):

$$
\operatorname{Re}\left(k_{S}\right)=0
$$

$$
\operatorname{Re}\left(k_{H}\right) \text { is positive definite Hermitian }
$$

$k=\left(k_{i j}\right)$ is positive definite and Hermitian if and only if

$$
k_{S}=0
$$

Remark: In particular any positive definite Hermitian $k=\left(k_{i j}\right)$ has the form

$$
k=k_{R}+i k_{I}
$$

where $k_{R}=\operatorname{Re}\left(k_{H}\right)$ is real PDH and $k_{I}=\operatorname{Im}\left(k_{H}\right)$ real Hermitian.

Proof. The decomposition (4.10) and the positive definiteness of $k$ imply that

$$
\begin{gathered}
0 \leq\langle x, k x\rangle=\left\langle x, \operatorname{Re}\left(k_{H}\right) x\right\rangle+\left\langle x, i \operatorname{Im}\left(k_{H}\right) x\right\rangle+\left\langle x, i \operatorname{Re}\left(k_{S}\right) x\right\rangle-\left\langle x, \operatorname{Im}\left(k_{S}\right) x\right\rangle \\
=\left\langle x, \operatorname{Re}\left(k_{H}\right) x\right\rangle+i\left\langle x, \operatorname{Re}\left(k_{S}\right) x\right\rangle
\end{gathered}
$$

In particular the right hand side is real and, by Lemma $(3), \operatorname{Re}\left(k_{H}\right) \in M_{d}(\mathbb{R})$ is symmetric, so that:

$$
\begin{gathered}
\left\langle x, \operatorname{Re}\left(k_{H}\right) x\right\rangle+i\left\langle x, \operatorname{Re}\left(k_{S}\right) x\right\rangle=\left\langle\operatorname{Re}\left(k_{H}\right) x, x\right\rangle-i\left\langle\operatorname{Re}\left(k_{S}\right) x, x\right\rangle=\left\langle x, \operatorname{Re}\left(k_{H}\right) x\right\rangle-i\left\langle x, \operatorname{Re}\left(k_{S}\right) x\right\rangle \\
\Leftrightarrow\left\langle x, \operatorname{Re}\left(k_{S}\right) x\right\rangle=-\left\langle x, \operatorname{Re}\left(k_{S}\right) x\right\rangle \Leftrightarrow\left\langle x, \operatorname{Re}\left(k_{S}\right) x\right\rangle=0
\end{gathered}
$$

But, since $\operatorname{Re}\left(k_{S}\right)$ is real Hermitian, it can be symplectic if and only if it is zero, i.e. (4.11) holds. In this case

$$
0 \leq\left\langle x, \operatorname{Re}\left(k_{H}\right) x\right\rangle
$$

which is (4.12). Finally $k=\left(k_{i j}\right)$ is positive definite and Hermitian if and only if

$$
\begin{gathered}
\operatorname{Re}\left(k_{H}\right)+i \operatorname{Im}\left(k_{H}\right)-\operatorname{Im}\left(k_{S}\right)=k=k^{*}=\operatorname{Re}\left(k_{H}\right)^{*}-i \operatorname{Im}\left(k_{H}\right)^{*}-\operatorname{Im}\left(k_{S}\right)^{*}= \\
=\operatorname{Re}\left(k_{H}\right)+i \operatorname{Im}\left(k_{H}\right)+\operatorname{Im}\left(k_{S}\right) \Leftrightarrow \operatorname{Im}\left(k_{S}\right)=0
\end{gathered}
$$

Lemma 7. Any positive definite matrix $k$ can be written in the form

$$
k=\operatorname{Re}\left(k_{H}\right)+i \operatorname{Im}\left(k_{H}\right)-\operatorname{Im}\left(k_{S}\right)
$$

where $\operatorname{Re}\left(k_{H}\right)$ is a real positive definite matrix and $\operatorname{Im}\left(k_{H}\right) \operatorname{Im}\left(k_{S}\right)$ are real skew-symmetric. Conversely if $\operatorname{Re}\left(k_{H}\right), \operatorname{Im}\left(k_{H}\right)$ and $\operatorname{Im}\left(k_{S}\right)$ have these properties, then the right hand side of (4.15) is a positive definite matrix.

Proof. Clear from Lemma (6). 


\section{Positive Definite Kernels}

Definition 4. A kernel $k(\cdot, \cdot)$ on $S$ is called positive definite if $\forall n \in \mathbb{N}, \forall x_{1}, \ldots, x_{n} \in S, \forall \xi_{1}, \ldots, \xi_{n} \in \mathbb{N}$

$$
\xi_{k}^{*} k\left(x_{k}, x_{j}\right) \xi_{j} \geq 0
$$

i.e. if the matrix $\left(k\left(x_{i}, x_{j}\right)\right)$ is positive definite.

Remark: From (5.1) it is clear that PD kernels on $S$ are a cone, closed under pointwise convergence.

Lemma 8. Given a kernel $k$ on $S$, define the kernels $\bar{k}, k^{*}$ respectively by

$$
\begin{aligned}
\bar{k}(x, y) & :=k(x, y)^{*} \\
k^{*}(x, y) & :=k(y, x)^{*}
\end{aligned}
$$

Then one of these kernels is $P D$ (resp. CPD) if and only if the other two are $P D$.

Proof.

$$
\xi_{j}^{*} k^{*}\left(x_{j}, x_{k}\right) \xi_{k}=\xi_{j}^{*} k\left(x_{k}, x_{j}\right)^{*} \xi_{k}=\left(\xi_{k}^{*} k\left(x_{k}, x_{j}\right) \xi_{j}\right)^{*} \geq 0 \Leftrightarrow \xi_{k}^{*} k\left(x_{k}, x_{j}\right) \xi_{j} \geq 0
$$

Thus $k$ is PD (resp. CPD) if and only if $k^{*}$ is. Similarly

$$
\begin{gathered}
\xi_{j}^{*} \bar{k}\left(x_{j}, x_{k}\right) \xi_{k}=\xi_{j}^{*} k\left(x_{j}, x_{k}\right)^{*} \xi_{k}=\left(\xi_{k}^{*} k\left(x_{j}, x_{k},\right) \xi_{j}\right)^{*}=\left(\xi_{j} k\left(x_{j}, x_{k},\right) \xi_{k}^{*}\right)^{*} \geq 0 \Leftrightarrow \\
\xi_{j} k\left(x_{j}, x_{k},\right) \xi_{k}^{*} \geq 0
\end{gathered}
$$

Thus $k$ is $\mathrm{PD}$ (resp. CPD) if and only if $\bar{k}$ is.

Corollary 1. If $k$ is a PD (resp. CPD) kernel then its real part defined by

$$
\operatorname{Re}(k)(x, y):=\frac{1}{2}\left(k(x, y)+k(y, x)^{*}\right)
$$

is $P D$.

Corollary 2. If $k(f, g)$ is a positive definite kernel on $S$ then, for any family $t_{f} \in \mathbb{C}$, also the kernel

$$
t_{f}^{*} k(f, g) t_{g}
$$

is positive definite. Conversely, if there exists a map

$$
c: f \in G \rightarrow c_{f} \in \mathbb{C} \backslash\{0\}
$$

(i.e. with all $c_{f}$ 's invertible) such that the kernel

$$
H(f, g):=c_{f}^{*} k(f, g) c_{g}
$$

is $P D$, then the kernel $k(f, g)$ itself is $P D$.

Proof. Let $k(f, g)$ be positive definite. Then, for any family $t_{f} \in \mathbb{C}$, also the kernel

$$
k_{t}(f, g):=t_{f}^{*} k(f, g) t_{g}=k(f, g) t_{f}^{*} t_{g}
$$

is PD, being the Schur product of two PD kernels. Conversely, if the kernel $H(f, g)$, defined by (5.4) is PD then, by the first part of the Lemma, also the kernel

$$
k(f, g)=\left(c_{f}^{-1}\right)^{*} H(f, g) c_{g}^{-1}
$$

is $\mathrm{PD}$.

Definition 5. Two positive definite kernels on $S, k_{1}, k_{2}$ are called equivalent if for some function $t: S \rightarrow$ $\mathbb{C} \backslash\{0\}$

$$
k_{1}(f, g)=t_{f}^{*} k_{2}(f, g) t_{g}
$$




\subsection{Schur's lemma.}

Lemma 9. (Schur) If $H=\left(H_{j k}\right), k=\left(k_{j k}\right)$ are PD then their Schur product

$$
H \circ k:=\left(H_{j k} k_{j k}\right)
$$

is $P D$. In particular the cone of PD kernels on $S$ is a semigroup under Schur multiplication with identity given by

$$
\begin{gathered}
1 \circ 1^{*} \quad ; \quad\left(1 \circ 1^{*}\right)_{i j}:=1 \quad ; \quad \forall i, j \in S \\
k=\sum_{m} \alpha_{m} x_{m} x_{m}^{*} \quad ; \quad k_{j k}:=\alpha_{m} x_{m i} \bar{x}_{m k} \\
H=\sum_{m} \beta_{m} x_{m} x_{m}^{*} ; \quad H_{j k}:=\beta_{m} x_{m i} \bar{x}_{m k} \\
\alpha_{m}, \beta_{n} \geq 0 \\
\bar{\lambda}_{j}(k \circ H)_{j k} \lambda_{k}=\bar{\lambda}_{j} k_{j k} H_{j k} \lambda_{k}=\bar{\lambda}_{j} \alpha_{m} x_{m j} \bar{x}_{m k} \beta_{n} y_{n j} \bar{y}_{n k} \lambda_{k} \\
=\alpha_{m} \beta_{m}\left(\bar{\lambda}_{j} x_{m j} y_{n j}\right)\left(\bar{x}_{m k} \bar{y}_{n k} \lambda_{k}\right)=\alpha_{m} \beta_{m}\left|\sum_{k} \bar{x}_{m k} y_{n k} \lambda_{k}\right|^{2} \geq 0
\end{gathered}
$$

Other proof:

$$
a=\left(a_{j k}\right) \quad: \quad b=\left(b_{j k}\right)
$$

By the spectral theorem for matrices

$$
\begin{gathered}
a=x^{*} x \quad: \quad b=y^{*} y \\
\bar{\lambda}_{i} a_{i k} b_{i k} \lambda_{k}=\bar{\lambda}_{i} x_{i j}^{*} x_{j k} y_{i l}^{*} y_{l k} \lambda_{k}=\bar{\lambda}_{i} \overline{x_{j i} y_{l i}} x_{j k} y_{l k} \lambda_{k}=\left|x_{j k} y_{l k} \lambda_{k}\right|^{2} \geq 0
\end{gathered}
$$

Lemma 10. The family of $\mathbb{C}$-valued positive definite matrices (kernels on $S$ ) is closed under:

- pointwise multiplication (Schur's Lemma)

- pointwise addition

- pointwise multiplication by a positive scalar

- pointwise limits

- integrals

Proof. From the definition of positive-definiteness.

Definition 6. A Borel function $f: \mathbb{C} \rightarrow \mathbb{C}$ is called completely positive (Hermitian) if $\forall d \in \mathbb{N}$ and for any positive definite (Hermitian) matrix $A=\left(a_{i j}\right)$ the matrix

$$
f \circ A:=\left(f\left(a_{i j}\right)\right)
$$

is positive definite (Hermitian)

Remark: The above definition is equivalent to say that, for any set $S$ and for any kernel $k$ on $S$, the composite kernel on $S$

$$
f \circ k=: f(k)
$$

is positive definite (Hermitian).

Remark: An interesting open problem is to characterize the completely positive (Hermitian) Borel functions $f: \mathbb{C} \rightarrow \mathbb{C}$.

Corollary 3. The exponential function on $\mathbb{C}$ is completely positive and Hermitian. 
Proof. We have to prove that, if $k$ is a positive definite Hermitian kernel on $S$, then its exponential kernel has these properties. By Schur's Lemma $\forall n \in \mathbb{N} k^{n}$ (Schur's power) is positive definite. Lemma (10) implies that $e^{k}$ is positive definite. Moreover $\forall x, y \in S$

$$
\left(e^{k(x, y)}\right)^{*}=e^{\overline{k(x, y)}}=e^{k(y, x)}
$$

Therefore $e^{k}$ is Hermitian.

Remark: In fact we will see that the exponential function on $\mathbb{C}$ has a much stronger positivity property (see Theorem (5) and Corollary (4)).

Definition 7. A kernel $h$ is called exponential if it is of the form $h=e^{k}$ where $k$ is a kernel on $S$. In this case $h$ is called the exponential kernel of $k$.

\section{Functions of positive Definite matrices}

Theorem 4. Let $A=\left(a_{i j}\right)$ be any Hermitian complex matrix. Then there exists an $\bar{\varepsilon}>0$ such that $\forall \varepsilon \in[-\bar{\varepsilon}, \bar{\varepsilon}]$

$$
1+\varepsilon A
$$

is invertible and both $1+\varepsilon A$ and its inverse are $P D$ and Hermitian.

Proof. Since, for any unitary matrix $U$, the properties of being PDH are invariant under the transformation $A \mapsto U^{*} A U$, we can suppose that $A$ is diagonal, say $A=\operatorname{diag}\left(s_{1}, \ldots, s_{n}\right)$, with the $s_{j}$ real. Then

$$
1+\varepsilon A=\operatorname{diag}\left(1+\varepsilon s_{1}, \ldots, 1+\varepsilon s_{n}\right)
$$

is Hermitian, $\mathrm{PD}$ and, for $\varepsilon$ small enough, invertible. For the same $\varepsilon$ :

$$
(1+\varepsilon A)_{i j}^{-1}=\delta_{i j}\left(1+\varepsilon s_{j}\right)^{-1} \geq 0
$$

Thus $(1+\varepsilon A)^{-1}$ is also positive and this proves the thesis.

Theorem 5. Let $\left(a_{i j}\right)$ be an Hermitian matrix. Then $\forall t \in \mathbb{R}$ the matrix $\left(e^{t a_{i j}}\right)$ is positive definite and Hermitian.

Proof. Let $\left(a_{i j}\right)$ be as in the statement and let $\bar{\varepsilon}>0$ be as in Theorem (4). Then, $\forall t \in \mathbb{R}$, the matrix $e^{t a_{i j}}$ is Hermitian and for all $n \in \mathbb{N}$ such that $|t / n|<\bar{\varepsilon}$, the matrix

$$
\left(1+\frac{t a_{i j}}{n}\right)^{n}
$$

is PD. It follows that

$$
\left(e^{t a_{i j}}\right)=\lim _{n \rightarrow \infty}\left(1+\frac{t a_{i j}}{n}\right)^{n}
$$

is PD and Hermitian.

Corollary 4. Let $\left(a_{i j}\right)$ be a positive definite Hermitian matrix. Then $\forall t \in \mathbb{R}$ the matrix $\left(e^{i t I m\left(a_{i j}\right)}\right)$ is positive definite and Hermitian.

Proof. Let $\left(a_{i j}\right)$ be a positive definite Hermitian matrix. From Lemma $(7)$ we know that $\left(\operatorname{Re}\left(a_{i j}\right)\right.$ is PD and Hermitian hence, Theorem (5) implies that, $\forall t \in \mathbb{R}$, both $\left(e^{-t R e\left(a_{i j}\right)}\right)$ and that $\left(e^{t a_{i j}}\right)$ are $\mathrm{PD}$ and Hermitian. Therefore $\forall t \in \mathbb{R}$

$$
\left(e^{t a_{i j}}\right)\left(e^{-t \operatorname{Re}\left(a_{i j}\right)}\right)=\left(e^{i t \operatorname{Im}\left(a_{i j}\right)}\right)
$$

is PD and Hermitian. 
Corollary 5. Let $\left(a_{i j}\right)$ be a positive definite Hermitian matrix such that

$$
\operatorname{Re}\left(a_{i j}\right)>0 \quad ; \quad \forall i, j
$$

Then

is a positive definite Hermitian matrix.

$$
\left(\frac{1}{a_{i j}}\right)
$$

Proof. By Theorem (4) $\left(e^{-t a_{i j}}\right)$ is PD Hermitian for any $t>0$ and condition (6.1) implies that $\forall i, j$ the function $t \in \mathbb{R}_{+} \mapsto e^{-t a_{i j}}$ is integrable. Therefore by Lemma (10) the matrix

$$
\int_{0}^{+\infty} e^{-t a_{i j}} d t=\int_{0}^{+\infty} \frac{d e^{-t a_{i j}}}{-a_{i j}}=\left.\frac{e^{-t a_{i j}}}{-a_{i j}}\right|_{0} ^{\infty}=\frac{1}{a_{i j}} \quad ; \quad \forall i, j
$$

is PD Hermitian as an integral of PD Hermitian matrices.

\section{FunCtions of POSitive DEFINITE KeRNELS}

Theorem 6. $\forall t \in \mathbb{R}$ the functions

$$
z \in \mathbb{C} \mapsto e^{t z} \quad ; \quad z \in \mathbb{C} \mapsto e^{i t \operatorname{Im}(z)}
$$

are completely positive and Hermitian.

Proof. Let $S$ be a set and let $k$ be a $\mathrm{PD}(\mathrm{H})$ kernel on $S$. Then, for any finite subset $F \subseteq S,(k(x, y))_{x, y \in F}$ is a $\mathrm{PD}(\mathrm{H})$ matrix. Hence, $\forall t \in \mathbb{R}$ the matrices

$$
e^{t k(x, y)} \quad ; \quad e^{i t I m(k(x, y))} \quad ; \quad x, y \in F
$$

are $\mathrm{PD}(\mathrm{H})$, i.e. the kernels

$$
e^{t k} \quad ; \quad e^{i t I m(k)}
$$

are $\mathrm{PD}(\mathrm{H})$ and this implies the thesis.

Corollary 6. A kernel of the form $h=e^{k}$ with $k$ positive definite Hermitian is Schur-invertible and its inverse is also PDH.

Proof. Since $k$ is positive definite Hermitian it follows that, $\forall t \in \mathbb{R}$, $e^{t k}$ is $\mathrm{PDH}$. In particular $e^{-k}=\left(e^{k}\right)^{-1}$ is PDH.

Corollary 7. If $K$ is a positive definite Hermitian kernel on $S$ then, for any $a<b \in \mathbb{R}_{+}$, the kernel on $S$

$$
e^{b K(x, y)}-e^{a K(x, y)}
$$

is positive definite Hermitian.

Remark: In particular the kernels

$$
\begin{gathered}
e^{K(x, y)}-1 \\
\sinh (t K(x, y)) \quad ; \quad \forall t \in \mathbb{R}
\end{gathered}
$$

are PD Hermitian (the PD and Hermitianity of $\cosh (t K(x, y))$ follows from Lemma (10).

Proof. The kernels $K(x, y)$ and $(\forall t \in \mathbb{R})$ are positive definite Hermitian. From this it follows that

$$
\int_{a}^{b} K(x, y) e^{t K(x, y)} d t=e^{b K(x, y)}-e^{a K(x, y)}
$$

is PD Hermitian. 
Remark: The analogue of Theorem (4) in general is false. In fact, if $K: S \times S \rightarrow \mathbb{C}$ is an Hermitian kernel on $S$, then for every finite subset $F \subseteq S$ there exists an $\bar{\varepsilon}_{F}>0$ such that, $\forall \varepsilon \in\left[-\bar{\varepsilon}_{F}, \bar{\varepsilon}_{F}\right]$ the kernel on $S$

$$
(1+\varepsilon K)(x, y):=\delta_{x, y}+\varepsilon K(x, y)
$$

is $\mathrm{PD}(\mathrm{H})$. The basic difference between the matrices (finite dimensional case) and kernels (infinite dimensional case) is that in the above $\bar{\varepsilon}_{F}$ depends on the finite set $F$. The result is true if $k$ is bounded in $L^{\infty}$-norm.

\section{Conditionally positive Definite Kernels}

Semigroups of positive definite matrices play an important role in several fields of mathematics. In this section we characterize their generators. We begin with some necessary conditions.

Lemma 11. If, $\forall t \in \mathbb{R}_{+}$the kernel $\left(e^{t k_{i j}}\right)$ is $P D$, then the kernel $\left(k_{i j}\right)$ is such that for any finite family of complex numbers $\left(\lambda_{j}\right)$ satisfying

$$
\sum_{j} \lambda_{j}=0
$$

one has:

$$
\sum_{j, k} \bar{\lambda}_{i} k_{i j} \lambda_{j} \geq 0
$$

Such a $k$ admits a unique representation of the form

$$
k=k_{0}+S
$$

with $k_{0}=k_{0}^{*}$ is positive definite and $S$ real symplectic.

In the representation (8.3), $S=0$ if and only if $\forall t \in \mathbb{R}_{+}$the kernel ( $\left.e^{t k_{i j}}\right)$ is $P D$ and Hermitian,

Proof. We begin proving (8.2). Suppose that $\forall t \in \mathbb{R}_{+}$the kernel $\left(e^{t k_{i j}}\right)$ is $\mathrm{PD}$, i.e. that $\forall t \in[0, T]$

$$
\bar{\lambda}_{i} e^{t k_{i j}} \lambda_{j} \geq 0
$$

then, whenever condition (8.1) is satisfied, one has, $\forall t \in[0, T]$, assuming summation over repeated indices:

$$
0 \leq \bar{\lambda}_{i} e^{t k_{i j}} \lambda_{j}=\bar{\lambda}_{i} e^{t k_{i j}} \lambda_{j}-\bar{\lambda}_{i} \lambda_{j}
$$

therefore, for $t \geq 0$ :

$$
0 \leq \bar{\lambda}_{i} \frac{\left(e^{t k_{i j}}-1\right)}{t} \lambda_{j}
$$

and, letting $t \downarrow 0,(8.2)$ follows.

Finally if, $\forall t \in \mathbb{R}_{+}$the kernel $\left(e^{t k_{i j}}\right)$ is Hermitian, then

$$
\left.\frac{d}{d t}\right|_{t=0}\left(e^{t k_{i j}}\right)^{*}=\left(\left.\frac{d}{d t}\right|_{t=0} e^{t k_{i j}}\right)^{*}=\overline{k_{i, j}}=\left.\frac{d}{d t}\right|_{t=0} e^{t k_{j, i}}=k_{j, i}
$$

i.e. $k$ is self-adjoint, which implies $S=0$.

To prove the hermitianity of the kernel $k=\left(k_{i j}\right)$ denote $\forall i, j \in S$

$$
k_{i j}=: \operatorname{Re}\left(k_{i j}\right)+i \operatorname{Im}\left(k_{i j}\right)=: k_{H, i j}+i k_{S, i j}=: k_{H}(i j)+i k_{S}(i j)
$$

with $k_{H, j j}, k_{S, j j} \in \mathbb{R}$. Since $\forall t \in \mathbb{R}_{+}$the kernel $\left(e^{t k_{i j}}\right)$ is PD, by a previous Remark,

$$
e^{t k_{j j}}=e^{t k_{H, j j}}\left(\cos \left(t k_{S, j j}\right)+i \sin \left(t k_{S, j j}\right) \geq 0 \quad ; \quad \forall j \in S ; \forall t \geq 0\right.
$$

which is possible if and only if $\forall t \geq 0$ and $\forall j \in X$

$$
\sin \left(t_{S, j j}\right)=0 \quad \Leftrightarrow \quad k_{S, j j}=0
$$


and this proves that

$$
k_{i i} \in \mathbb{R} \quad ; \quad \forall i \in S
$$

Finally condition (8.2) implies in particular that $\forall \lambda \in \mathbb{C}$ and $\forall i, j \in X$

$$
0 \leq \bar{\lambda} k(i, i) \lambda+\lambda k(j, j) \bar{\lambda}-\bar{\lambda} k(i, j) \lambda-\bar{\lambda} k(j, i) \lambda
$$

and, since $k_{S}(i, i)=0$ because of (8.6), putting $\lambda=1$, this becomes

$$
0 \leq k(i, i)+k(j, j)-k(i, j)-k(j, i)=k_{H}(i, i)+k_{H}(j, j)-k_{H}(i, j)-k_{H}(i, j)-i\left(k_{S}(i, j)+k_{S}(j, i)\right)
$$

Thus

$$
k_{S}(i, j)+k_{S}(j, i)=0 \Leftrightarrow k_{S}(i, j)=-k_{S}(j, i)
$$

Thus we can write

$$
k_{i j}=k_{H, i, j}+i k_{S, i, j}=\frac{1}{2}\left(k_{H, i, j}+k_{H, j, i}\right)+\frac{1}{2}\left(k_{H, i, j}-k_{H, j, i}\right)+i k_{S, i, j}
$$

and defining

$$
\begin{aligned}
& k_{0, i, j}:= \frac{1}{2}\left(k_{H, i, j}+k_{H, j, i}\right)+i k_{S, i, j}=\overline{k_{0, j, i}} \\
& S_{i, j}:=\frac{1}{2}\left(k_{H, i, j}-k_{H, j, i}\right)
\end{aligned}
$$

we see that $k_{0}$ is self-adjoint, $S$ real symplectic and (8.3) is satisfied. If $k=k_{0}^{\prime}+S^{\prime}$ is another representation of the form (8.3), then

This implies that

$$
k+S=k_{0}^{\prime}+S^{\prime} \Leftrightarrow k-k_{0}^{\prime}=S^{\prime}-S
$$

$$
\begin{gathered}
\operatorname{Im}\left(k-k_{0}^{\prime}\right)=0 \Leftrightarrow \operatorname{Im}(k)=\operatorname{Im}\left(k_{0}^{\prime}\right) \\
\operatorname{Re}\left(k-k_{0}^{\prime}\right)=S^{\prime}-S
\end{gathered}
$$

But the left hand side is symmetric and the right hand side is anti-symmetric. Hence both sides must be zero and the representation (8.3) is unique.

Definition 8. In the notations of Lemma (11) a complex matrix $\left(k_{i j}\right) \in M_{d}(\mathbb{C})$ is said to be conditionally positive definite (CPD) if it satisfies the necessary conditions of Lemma (11), i.e. it has the form (8.3) and

$$
\sum_{j, k} \bar{\lambda}_{i} k_{i j} \lambda_{j} \geq 0
$$

for any finite family of complex numbers $\left(\lambda_{j}\right)$ such that

$$
\sum_{j} \lambda_{j}=0
$$

If in addition $k$ is Hermitian, we say that $k$ is CPD Hermitian.

Remark: From (8.3) it follows that, if $k$ is CPD then its diagonal elements are real.

Remark: If $f: S \rightarrow \mathbb{C}$ is any function, the kernels

$$
k(x, y):=f(x)+f(y) \quad ; \quad f(x)-f(y)
$$

are CPD and the kernel

$$
k(x, y):=f(x)+\overline{f(y)}
$$

is CPD Hermitian. All these kernels are called trivial.

Definition 9. A kernel $k(\cdot, \cdot)$ on $E$ is called conditionally positive definite if $\forall n \in \mathbb{N}, \forall x_{1}, \ldots, x_{n}$, the matrix $\left(k\left(x_{i}, x_{j}\right)\right)$ is conditionally positive definite. 
Lemma 12. Let $k$ be a CPD kernel on $X$ and, for any pair of functions $\alpha, \beta: X \rightarrow \mathbb{C}$, define the kernel

$$
h(x, y):=k(x, y)+\alpha(x)+\beta(y)
$$

Then the kernel (8.10):

(i) always satisfies condition (8.8) for any finite family of complex numbers $\left(\lambda_{j}\right)$ satisfying (8.9)

(ii) is CPD if and only if there exist real valued functions

$$
a, b, \gamma: x \in S \rightarrow a(x), b(x), \gamma(x) \in \mathbb{R}
$$

such that

$$
\alpha(x)=a(x)-i \gamma(x) \quad ; \quad \beta(x)=b(x)+i \gamma(x)
$$

(iii) is CPD Hermitian if and only if $S$ is trivial

(iv) if $k$ is Hermitian, $h$ is Hermitian if and only if for some constant $c \in \mathbb{R}$ one has

$$
\beta(x)=\bar{\alpha}(x)+c
$$

In this case, denoting

$$
\alpha_{0}(x):=\frac{1}{2} c+\alpha(x)
$$

one has

$$
h(x, y):=k(x, y)+\alpha_{0}(x)+\overline{\alpha_{0}}(y)
$$

and $h$ is $C P D$ if and only if $k$ is.

Proof. (i) It is clear that, if $k$ is CPD, then for any choice of the functions $\alpha$ and $\beta$, the right hand side of (8.10) satisfies condition (8.8) for any finite family of complex numbers $\left(\lambda_{j}\right)$ satisfying (8.9).

(ii) From the decomposition (8.3) of $k$ one deduces that, for $h$ to be CPD, one must have the identity

$$
h(x, y)=k_{0}(x, y)+S(x, y)+\alpha(x)+\beta(y)=h_{0}(x, y)+S_{0}(x, y)
$$

for some $h_{0}=h_{0}^{*}$ and $S_{0}$ real symplectic. This is equivalent to

$$
k_{0}(x, y)-h_{0}(x, y)=S_{0}(x, y)-S(x, y)-\alpha(x)-\beta(y)
$$

Taking adjoints this gives

$$
\overline{k_{0}(x, y)}-\overline{h_{0}(x, y)}=S_{0}(x, y)-S(x, y)-\overline{\alpha(x)}-\overline{\beta(y)}
$$

Since $h_{0}$ and $k_{0}$ are Hermitian, this implies that

$$
\begin{gathered}
S_{0}(x, y)-S(x, y)-\overline{\alpha(x)}-\overline{\beta(y)}=\overline{k_{0}(x, y)}-\overline{h_{0}(x, y)}=k_{0}(y, x)-h_{0}(y, x)= \\
=S_{0}(y, x)-S(y, x)-\alpha(y)-\beta(x)=-S_{0}(x, y)+S(x, y)-\alpha(y)-\beta(x) \Leftrightarrow \\
\Leftrightarrow 2 S_{0}(x, y)-2 S(x, y)=\overline{\alpha(x)}-\alpha(y)+\overline{\beta(y)}-\beta(x)
\end{gathered}
$$

exchanging the roles of $x$ and $y$ one has

$$
2 S_{0}(y, x)-2 S(y, x)=2 S(x, y)-2 S_{0}(x, y)=\overline{\alpha(y)}-\alpha(x)+\overline{\beta(x)}-\beta(y)
$$

Thus the right hand sides of the two equations must be opposite:

$$
\begin{gathered}
\overline{\alpha(x)}-\alpha(y)+\overline{\beta(y)}-\beta(x)=-\overline{\alpha(y)}+\alpha(x)-\overline{\beta(x)}+\beta(y) \Leftrightarrow \\
\Leftrightarrow 0=(\alpha(y)-\overline{\alpha(y)})+(\alpha(x)-\overline{\alpha(x)})+(\beta(x)-\overline{\beta(x)})+(\beta(y)-\overline{\beta(y)})
\end{gathered}
$$

Putting $x=y$ gives

$$
\begin{gathered}
0=2(\alpha(x)-\overline{\alpha(x)})+2(\beta(x)-\overline{\beta(x)})=4 i \operatorname{Im}(\alpha(x))+4 i \operatorname{Im}(\beta(x)) \Leftrightarrow \\
\Leftrightarrow \operatorname{Im}(\beta(x))=-\operatorname{Im}(\alpha(x))=:-\gamma(x)
\end{gathered}
$$




$$
\Leftrightarrow \alpha(x))=a(x)-i \gamma(x) \quad ; \quad \beta(x)=b(x)-i \gamma(x) \quad ; \quad a(x), b(x), \gamma(x) \in \mathbb{R}
$$

and this proves (8.12). Conversely, if (8.11) and (8.12) are satisfied, then

$$
\begin{gathered}
h(x, y)=k_{0}(x, y)+a(x)+b(y)+i(\gamma(y)-\gamma(x))+S(x, y) \\
=k_{0}(x, y)+\frac{1}{2}(a(x)+a(y))+\frac{1}{2}(b(y)+b(x))+i(\gamma(y)-\gamma(x))+\frac{1}{2}(a(x)-a(y))+\frac{1}{2}(b(y)-b(x))+S(x, y)
\end{gathered}
$$

Thus defining

$$
\begin{gathered}
h_{0}(x, y):=k_{0}(x, y)+\frac{1}{2}(a(x)+a(y))+\frac{1}{2}(b(y)+b(x))+i(\gamma(y)-\gamma(x)) \\
S_{0}(x, y):=\frac{1}{2}(a(x)-a(y))+\frac{1}{2}(b(y)-b(x))+S(x, y)
\end{gathered}
$$

one has

$$
\begin{gathered}
\overline{h_{0}(x, y)}:=\overline{k_{0}(x, y)}+\frac{1}{2}(a(x)+a(y))+\frac{1}{2}(b(y)+b(x))-i(\gamma(y)-\gamma(x))= \\
=k_{0}(y, x)+\frac{1}{2}(a(y)+a(x))+\frac{1}{2}(b(x)+b(y))+i(\gamma(x)-\gamma(y))=h_{0}(y, x) \\
S_{0}(y, x):=\frac{1}{2}(a(y)-a(x))+\frac{1}{2}(b(x)-b(y))+S(y, x) \\
=-\frac{1}{2}(a(x)-a(y))-\frac{1}{2}(b(y)-b(x))-S(x, y)=-S_{0}(x, y)
\end{gathered}
$$

hence $h_{0}$ is Hermitian and $S_{0}$ is symplectic, so that $h$ is CPD.

(iii) is CPD Hermitian if and only if

$$
\begin{aligned}
& 0=S_{0}(x, y):=\frac{1}{2}(a(x)-a(y))+\frac{1}{2}(b(y)-b(x))+S(x, y) \Leftrightarrow \\
& \Leftrightarrow S(x, y)=\frac{1}{2}(a(x)-b(x))-\frac{1}{2}(a(y)-b(y))=: f(x)-f(y)
\end{aligned}
$$

i.e. $S$ is trivial.

(iv) $h$ is Hermitian if and only if

$$
\overline{h(x, y)}=h(y, x)
$$

Given (8.10) this is equivalent to

$$
\overline{k(x, y)}+\bar{\alpha}(x)+\bar{\beta}(y)=k(y, x)+\alpha(y)+\beta(x)
$$

If $k$ is Hermitian, this is equivalent to

$$
\bar{\alpha}(x)+\bar{\beta}(y)=\alpha(y)+\beta(x) \Leftrightarrow \bar{\alpha}(x)-\beta(x)=\alpha(y)-\bar{\beta}(y)
$$

Since $x, y \in S$ are arbitrary, there exists a constant $a \in \mathbb{C}$ such that

$$
\bar{\alpha}(x)-\beta(x)=: a
$$

is independent of $x \in S$. Moreover, from (8.16) with $x=y$, we see that $a$ must be real. Therefore

$$
\beta(x)=\bar{\alpha}(x)-a
$$

which is (8.13) with $c=-a$. Therefore $h$ can be written in the form (8.14) and from this it is clear that $h$ is CPD if and only if $k$ is. 
Theorem 7. Let $X$ be a set, $k$ a kernel on $X$ and $x_{0} \in X$ an element of $X$. Then the following statements are equivalent:

i) $k$ is conditionally positive definite and

$$
k\left(x_{0}, x_{0}\right) \leq 0
$$

ii) the kernel

$$
\langle x, y\rangle:=k(x, y)-k\left(x, x_{0}\right)-k\left(x_{0}, y\right)
$$

is positive definite on $X$.

Proof. For any finite set $F \subseteq S$ and any choice of $\lambda_{x} \in \mathbb{C}(x \in F)$ define

$$
\lambda_{x_{0}}:=-\sum_{x \in F} \lambda_{x}
$$

so that

Now consider the quantity

$$
\sum_{x \in F \cup\left\{x_{0}\right\}} \lambda_{x}=0
$$

$$
\begin{gathered}
=\sum_{x, y \in F} \bar{\lambda}_{x} k(x, y) \lambda_{y}-\left(\sum_{x} \lambda_{x}\right)^{-} \sum_{y \in F} k\left(x_{0}, y\right) \lambda_{y}-\sum_{x \in F} \bar{\lambda}_{x} k\left(x, x_{0}\right)\left(\sum_{x} \lambda_{y}\right) \\
=\sum_{x, y \in F} \bar{\lambda}_{x} k(x, y) \lambda_{y}+\bar{\lambda}_{x_{0}} \sum_{y \in F} k\left(x_{0}, y\right) \lambda_{y}+\sum_{x \in F} \bar{\lambda}_{x} k\left(x, x_{0}\right) \lambda_{x_{0}}= \\
=\sum_{x, y \in F} \bar{\lambda}_{x} k(x, y) \lambda_{y}+\bar{\lambda}_{x_{0}} \sum_{y \in F} k\left(x_{0}, y\right) \lambda_{y}+\sum_{x \in F} \bar{\lambda}_{x} k\left(x, x_{0}\right) \lambda_{x_{0}}+\bar{\lambda}_{x_{0}} k\left(x_{0}, x_{0}\right) \lambda_{x_{0}}-\bar{\lambda}_{x_{0}} k\left(x_{0}, x_{0}\right) \lambda_{x_{0}}= \\
=\sum_{x, y \in F \cup\left\{x_{0}\right\}} \bar{\lambda}_{x} k(x, y) \lambda_{y}-\bar{\lambda}_{x_{0}} k\left(x_{0}, x_{0}\right) \lambda_{x_{0}}
\end{gathered}
$$

If $k$ is conditionally positive definite, then this is

$$
\geq-\bar{\lambda}_{x_{0}} k\left(x_{0}, x_{0}\right) \lambda_{x_{0}}
$$

and if (8.18) holds, this is $\geq 0$.

Conversely, if the kernel (8.19) is PD, then the quantity (8.21) is positive for any finite set $F \subseteq S$ and any choice of $\lambda_{x} \in \mathbb{C}(x \in F)$. In particular, if the $\lambda_{x} \in \mathbb{C}$ are chosen so that

$$
\sum_{x \in F} \lambda_{x}=0
$$

then the identity (8.21) gives

$$
\begin{gathered}
0 \leq \sum_{x, y \in F} \bar{\lambda}_{x}\left(k(x, y)-k\left(x, x_{0}\right)-k\left(x_{0}, y\right)\right) \lambda_{y}= \\
=\sum_{x, y \in F} \bar{\lambda}_{x} k(x, y) \lambda_{y}-\left(\sum_{x} \lambda_{x}\right)^{-} \sum_{y \in F} k\left(x_{0}, y\right) \lambda_{y}-\sum_{x \in F} \bar{\lambda}_{x} k\left(x, x_{0}\right)\left(\sum_{x} \lambda_{y}\right)= \\
=\sum_{x, y \in F} \bar{\lambda}_{x} k(x, y) \lambda_{y}
\end{gathered}
$$

i.e. the kernel $k(x, y)$ is CPD. 
Finally, if the kernel (8.19) is $\mathrm{PD}$, then choosing $x_{0}=x=y$ one finds:

$$
0 \leq\left\langle x_{0}, x_{0}\right\rangle=k\left(x_{0}, x_{0}\right)-k\left(x_{0}, x_{0}\right)-k\left(x_{0}, x_{0}\right)=-k\left(x_{0}, x_{0}\right)
$$

which is equivalent to (8.18).

Theorem 8. The following statements are equivalent::

(i) There exists an interval $[0, T]$ such that $\forall t \in[0, T]$ the kernel $\left(e^{t k_{i j}}\right)$ is $P D$

(ii) $\forall t \in \mathbb{R}_{+}$the kernel $\left(e^{t k_{i j}}\right)$ is $P D$

(iii) the kernel $\left(k_{i j}\right)$ is $C P D$

Proof. (i) $\Rightarrow$ (ii). By Schur's theorem, $\forall s, t \in[0, T]$ the kernel

$$
e^{s k_{i j}} e^{t k_{i j}}=e^{(s+t) k_{i j}}
$$

is $\mathrm{PD}$ and from this (ii) follows.

Since clearly (ii) $\Rightarrow$ (i), it follows that (i) $\Leftrightarrow$ (ii).

(ii) $\Rightarrow$ (iii). This is the content of Lemma (11).

(iii) $\Rightarrow$ (i) (in fact we will prove that (iii) $\Rightarrow\left(\right.$ ii)). Suppose that $\left(k_{i j}\right)$ is CPD. Then, by a previous Remark, its diagonal elements are real. Therefore one can find a function $\alpha,: i \in X \rightarrow \alpha_{i} \in \mathbb{C}$ such that the kernel

$$
h_{i j}:=k_{i j}-\alpha_{i}-\bar{\alpha}_{j}
$$

which is CPD by Lemma (12), satisfies

$$
h_{i i}=k_{i i}-\alpha_{i}-\bar{\alpha}_{i}=k_{i i}-2 \operatorname{Re}\left(\alpha_{i}\right) \leq 0 \quad ; \quad \forall i \in S
$$

By Theorem (7) for any $i_{0} \in X$ the kernel

$$
\begin{aligned}
h_{i j}-h_{i i_{0}} & -h_{i_{0} j}=k_{i j}-\alpha_{i}-\bar{\alpha}_{j}-k_{i i_{0}}+\alpha_{i}+\bar{\alpha}_{i_{0}}-k_{i_{0} j}+\alpha_{i_{0}}+\bar{\alpha}_{j} \\
& =k_{i j}-k_{i i_{0}}-k_{i_{0} j}+\alpha_{i_{0}}+\bar{\alpha}_{i_{0}}=: k_{i j}-\beta_{i}+\bar{\beta}_{j}
\end{aligned}
$$

with

$$
\beta_{i}:=k_{i i_{0}}-\alpha_{i_{0}}
$$

is PD. Therefore, by Theorem (4) $\forall t \geq 0$

$$
e^{t\left(k_{i j}-\beta_{i}-\bar{\beta}_{j}\right)}=e^{-t \beta_{i}} e^{t k_{i j}} e^{-t \bar{\beta}_{j}}=e^{-t \beta_{i}} e^{t k_{i j}}\left(e^{-t \bar{\beta}_{j}}\right)^{*}
$$

is PD hence, by Corollary (2), also

is PD $\forall t \geq 0$ and this proves (ii) hence (i).

Lemma 13. For two Hermitian Kernels $h, k$ the following are equivalent:

(i) $\forall u_{0} \in X$ the positive definite kernels

$$
k(u, v)-k\left(u, u_{0}\right)-k\left(u_{0}, v\right) \quad ; \quad h(u, v)-h\left(u, u_{0}\right)-h\left(u_{0}, v\right)
$$

differ by an additive real constant $c$.

(ii) There exists a function $\alpha: X \rightarrow \mathbb{C}$ such that

$$
h(u, v)=k(u, v)+\alpha(u)+\overline{\alpha(v)}+c
$$


Proof. If the kernels (8.22) differ by an additive real constant $c$, then $\forall u, u_{0}, v \in X$

$$
\begin{gathered}
k(u, v)-k\left(u, u_{0}\right)-k\left(u_{0}, v\right)=h(u, v)-h\left(u, u_{0}\right)-h\left(u_{0}, v\right)+c \\
\Leftrightarrow(k-h)(u, v)=(k-h)\left(u, u_{0}\right)+(k-h)\left(u_{0}, v\right)+c
\end{gathered}
$$

defining

$$
\alpha(u):=(k-h)\left(u, u_{0}\right)
$$

the Hermitianity of $h$ and $k$ implies that

$$
\overline{\alpha(v)}:=(k-h)\left(u_{0}, v\right)
$$

Conversely let $h$ be of the form

$$
h(u, v)=k(u, v)+\alpha(u)+\overline{\alpha(v)}+c
$$

then

$$
\begin{gathered}
h(u, v)-h\left(u, u_{0}\right)-h\left(u_{0}, v\right)= \\
k(u, v)+\alpha(u)+\overline{\alpha(v)}+c-k\left(u, u_{0}\right)-\alpha(u)-\overline{\alpha\left(u_{0}\right)}-c-k\left(u_{0}, v\right)-\alpha\left(u_{0}\right)-\overline{\alpha(v)}-c= \\
=k(u, v)-k\left(u, u_{0}\right)-k\left(u_{0}, v\right)-\overline{\alpha\left(u_{0}\right)}-\alpha\left(u_{0}\right)-c \\
=k(u, v)+k\left(u, u_{0}\right)+k\left(u_{0}, v\right)-\operatorname{Re}\left(\alpha\left(u_{0}\right)\right)+c
\end{gathered}
$$

i.e. the positive definite kernels (8.22) differ by an additive real constant $c$.

\section{INFINITELY DIVISIBLE KERNELS}

Definition 10. A (positive definite) kernel

$$
k: X \times X \rightarrow \mathbb{C}
$$

is called infinitely divisible, if $\forall n \in \mathbb{N}$ there exists a (positive definite) kernel $k_{n}$ such that

$$
k_{n}^{n}=k
$$

where $k_{n}^{n}$ denotes the $n-$ th Schur power of $k_{n}$

Lemma 14. For a PD kernel the following statements are equivalent:

(i) $k$ is infinitely divisible

(ii) $\forall n \in \mathbb{N}$ and $\forall x, y \in X$ there exists a choice of the $n$-th root of $k(x, y)$ such that the kernel

$$
k^{1 / n}(x, y):=(k(x, y))^{1 / n}
$$

is $P D$ (iii) For each $t \geq 0$ the kernel

is $P D$.

$$
k^{t}(x, y):=k(x, y)^{t}
$$

Proof. By definition if $k$ is infinitely divisible then for each $n$ and for some PD kernel $k_{n}^{n}$,

$$
k_{n}^{n}=k \Leftrightarrow k_{n}(i, j)^{n}=k(i, j) \quad ; \quad \forall i, j
$$

Therefore, denoting $\log (k(i, j))$ the principal determination of the logarithm of $k(i, j)$ one has

$$
\log \left(k_{n}(i, j)\right)=(1 / n) \log (k(i, j))
$$

Thus, defining

$$
k(i, j)^{1 / n}:=e^{(1 / n) \log (k(i, j))}
$$

one has

hence $k(i, j)^{1 / n}$ is PD. Therefore for each $k \in \mathbb{N}$

$$
k_{n}(i, j)=k(i, j)^{1 / n}
$$

$$
k(i, j)^{k / n}
$$


is also PD. Finally, defining $k^{t}$ by continuity, one has that $k^{t}$ is $\mathrm{PD} \forall t \geq 0$.

\section{The Kolmogorov decomposition theorem for $\mathbb{C}$-Valued PD Kernels}

Remark: The following theorem shows that every positive definite kernel can be seen as a scalar product in some Hilbert space.

Definition 11. A pre-Hilbert space is a vector space $\mathcal{H}$, over the real or complex numbers, with an antilinear embedding into its algebraic dual $\mathcal{H}^{*}$

$$
\xi \in \mathcal{H} \mapsto \xi^{*} \in \mathcal{H}^{*}
$$

such that the kernel on $\mathcal{H}$ defined by

$$
\langle\xi, \eta\rangle:=\xi^{*}(\eta)
$$

is positive definite.

Theorem 9. (Kolmogorov decomposition for positive definite kernels) Let $X$ be a set. If $k$ is a $\mathbb{C}$-valued positive definite kernel on $X$, then there exists an Hilbert space $\mathcal{H}$ and a map

$$
v: X \rightarrow \mathcal{H}
$$

such that:

$$
\begin{gathered}
k(x, y)=\langle v(x), v(y)\rangle=v(x)^{*} v(y) \quad ; \quad \forall x, y \in X \\
\{v(x): x \in X\} \quad \text { is total in } \mathcal{H}
\end{gathered}
$$

If $\left\{v_{0}, \mathcal{H}_{0}\right\}$ is another pair with these properties, then there exists a unitary operator $U: \mathcal{H} \rightarrow \mathcal{H}_{0}$ such that $U v=v_{0}$.

In particular a positive definite kernel on $S$ always satisfies the inequality

$$
|K(f, g)|^{2} \leq K(f, f) K(g, g)
$$

Proof. The atomic measures $\left\{\delta_{x}: x \in X\right\}$ are linearly independent probability measures on $X$ hence the map

$$
\left(\delta_{x}, \delta_{y}\right) \mapsto k(x, y)
$$

uniquely extends, by sesquilinearity, to a pre-scalar product on the complex vector space $\tilde{X}$ generated by them. Denote $\mathcal{H}$ the completion of the pre-Hilbert space obtained in this way and

$$
\eta: \tilde{X} \rightarrow \mathcal{H}
$$

the quotient map. Denote $v$ the restriction of $\eta$ on $X$. Then the map $v: X \rightarrow \mathcal{H}$ satisfies

$$
\langle v(x), v(y)\rangle=\left\langle\eta\left(\delta_{x}\right), \eta\left(\delta_{y}\right)\right\rangle=k(x, y)
$$

The remaining statements are clear.

Definition 12. A pair $\{v, H\}$, defined by Theorem (9), is called a Kolmogorov representation of the kernel $K: X \times X \rightarrow \mathbb{C}$. 


\section{BOSON FOCK SPACES}

Let $\mathcal{H}$ be an Hilbert space. Its scalar product $\langle\cdot, \cdot\rangle$ is a positive definite kernel on $\mathcal{H}$ hence, by Theorem $(4)$, also the kernel $\exp \langle\cdot, \cdot\rangle$ is.

Definition 13. The Kolmogorov decomposition of the $P D$ kernel $\exp \langle\cdot, \cdot\rangle$ on $\mathcal{H}$ is denoted

$$
\left\{e^{\mathcal{H}}, v=e^{\cdot}\right\}
$$

and called the exponential space (or Boson Fock space over $\mathcal{H}$ ).

The total set

is called the set of exponential vectors of $e^{\mathcal{H}}$.

$$
\exp (\mathcal{H}):=v(\mathcal{H}):=\left\{e^{f}=v_{f} \in e^{\mathcal{H}}: f \in \mathcal{H}\right\}
$$

Remark: Recall that by definition of Kolmogorov decomposition the characterizing property of the exponential vectors is

$$
\left\langle e^{f}, e^{g}\right\rangle=\left\langle v_{f}, v_{g}\right\rangle=e^{\langle f, g\rangle} \quad ; \quad \forall f, g \in \mathcal{H}
$$

\section{Infinitely DIVISIBLE KERNELS AND Boson FoCK SPACES}

Definition 14. Let $S$ be a set. A kernel $k$ on $S$ is called exponentially $P D$ if there exists a PD kernel $q$ such that $k$ is equivalent to $e^{q}$, i.e. if there exists a function $c: f \in S \rightarrow c_{f} \in \mathbb{C} \backslash\{0\}$ such that

$$
c_{f}^{*} k(f, g) c_{g}=e^{q(f, g)}=e^{q(f, g)-\kappa_{f}^{*}-\kappa_{g}} \quad ; \quad f, g \in S
$$

Theorem 10. For a kernel $k$ on a set $S$ the following statements are equivalent.

(i) $k(f, g)$ is exponentially $P D$

(ii) there exists a CPD kernel $q_{0}$ such that $k$ has the form

$$
k(f, g)=e^{q_{0}(f, g)} \quad ; \quad f, g \in S
$$

(iii) $k(f, g)$ is infinitely divisible, i.e. $k(f, g)^{t}$ is $P D, \forall t \geq 0$

(iv) there exists a PD kernel $q$ on $S$ and a map $f \in S \mapsto \kappa_{f} \in \mathbb{C}$ such that, denoting $\{\mathcal{H}, v\}$ (resp. $\{\mathcal{K}, u\}$ ) the Kolmogorov decomposition of $k$ (resp. q), then the map

$$
e^{\kappa_{f}} v_{f} \in \mathcal{H} \mapsto e^{u_{f}} \in \Gamma(\mathcal{K})
$$

extends to a unitary isomorphism between $\mathcal{H}$ and the Fock space $\Gamma(\mathcal{K})$ over $\mathcal{K}$.

Proof. (i) $\Rightarrow$ (ii). If $k$ is an exponentially PD kernel then it has the form $(12.1)$ with $q(\cdot, \cdot)$ PD. Therefore, defining

$$
\kappa_{f}:=\log _{f}
$$

where $\log c_{f}$ denotes the principal determination of the logarithm of $c_{f}$ one has

$$
c_{f}=: e^{\kappa_{f}}
$$

and the identity (12.6) holds with

$$
q_{0}(f, g):=q(f, g)-\kappa_{f}^{*}-\kappa_{g}
$$

From Lemma (12) we know that the kernel $q_{0}(f, g)$ is CPD because $q(f, g)$ is PD hence a fortiori CPD. (ii) $\Leftrightarrow\left(\right.$ iii). $k(f, g)$ has the form (12.6), for some CPD kernel $q_{0}$, if and only if $\forall t \geq 0$

$$
k(f, g)^{t}=e^{t q_{0}(f, g)}
$$


and, since $q_{0}(f, g)$ is $\mathrm{CPD}$, the right and side is $\mathrm{PD}$ by Theorem (8). This is equivalent to say that $k(f, g)$ is infinitely divisible.

(iii) $\Rightarrow$ (iv) If $k$ is infinitely divisible then, by Theorem (8), it has the form (12.6) for some CPD kernel $q_{0}$. Then by Theorem (7), and up to replacing $q_{0}$ by an equivalent CPD Hermitian kernel, $\forall f_{0} \in S$ the kernel

$$
q_{1}(f, g):=q_{0}(f, g)-q_{0}\left(f, f_{0}\right)-q_{0}\left(f_{0}, g\right)
$$

is $\mathrm{PD}$.

By construction the Kolmogorov decomposition $\{\mathcal{H}, v\}$, of $k$, satisfies

$$
\left\langle v_{f}, v_{g}\right\rangle=k(f, g)=e^{q_{0}(f, g)}
$$

Therefore

$$
\begin{gathered}
\left\langle e^{-q_{0}\left(f, f_{0}\right)^{*}} v_{f}, e^{-q_{0}\left(f_{0}, g\right)} v_{g}\right\rangle=\left\langle e^{-q_{0}\left(f_{0}, f\right)} v_{f}, e^{-q_{0}\left(f_{0}, g\right)} v_{g}\right\rangle= \\
=e^{-q_{0}\left(f, f_{0}\right)} e^{q_{0}(f, g)} e^{-q_{0}\left(f_{0}, g\right)}=e^{-q_{1}(f, g)}
\end{gathered}
$$

and (iv) follows by choosing

$$
\kappa_{f}:=q_{0}\left(f_{0}, f\right)
$$

(iv) $\Leftrightarrow$ (i). Suppose that (iv) holds and define

$$
c_{f}:=e^{\kappa_{f}}
$$

the unitary isomorphism (12.3) implies that

$$
c_{f}^{*} k(f, g) c_{g}=\left\langle e^{\kappa_{f}} v_{f}, e^{\kappa_{g}} v_{g}\right\rangle=\left\langle e^{u_{f}}, e^{u_{g}}\right\rangle=e^{\left\langle u_{f}, u_{g}\right\rangle}=e^{q\left(u_{f}, u_{g}\right)}
$$

this means that $k(f, g)$ is exponentially $\mathrm{PD}$, i.e. (i) holds.

Theorem 11. Let $S$ be a set. The infinitely divisible Hermitian kernels on $S$ are a group under pointwise multiplication.

Proof. According to Theorem (10) (ii), $k$ is an infinitely divisible Hermitian kernel on $S$ if and only if on $S$ there exists a CPDH kernel $q_{0}$ such that $k$ has the form

$$
k(f, g)=e^{q_{0}(f, g)} \quad ; \quad f, g \in S
$$

Up to replacing $q_{0}$ by an equivalent $\mathrm{CPDH}$ kernel one can assume that there exists $f_{0} \in S$ such that

$$
q_{0}\left(f_{0}, f_{0}\right)=0
$$

In fact, for any $f_{0} \in S$, if $\alpha: \mathbb{C} \rightarrow \mathbb{C}$ is any function such that

then the CPDH kernel defined by

$$
q_{0}\left(f_{0}, f_{0}\right)=2 \operatorname{Re}\left(\alpha_{f_{0}}\right)
$$

by construction is equivalent to $q_{0}$ and such that $q_{0}^{\prime}\left(f_{0}, f_{0}\right)=0$.

By Theorem (7) the kernel

$$
q(f, g):=q_{0}(f, g)-q_{0}\left(f, f_{0}\right)-q_{0}\left(f_{0}, g\right)
$$

is $\mathrm{PDH}$ hence, by Theorem (6), for any $t \in \mathbb{R}$, the kernel $e^{t q}$ is $\mathrm{PDH}$. In particular $e^{-q}$, which is the pointwise inverse of $e^{q}$, is $\mathrm{PDH}$ and for any $t \in \mathbb{R}_{+}$one has

$$
\left(e^{-q}\right)^{t}=e^{-t q}
$$

so that the kernel $\left(e^{-q}\right)^{t}$ is $\mathrm{PDH}$, i.e. the kernel $e^{-q}$ is infinitely divisible. 


\section{REFERENCES}

[1] Accardi, L., Boukas, A.: Random variables and positive definite kernels associated with the Schroedinger algebra, Proceedings of the VIII International Workshop Lie Theory and its Applications in Physics, Varna, Bulgaria, June 16-21, 2009, pages 126-137, American Institute of Physics , AIP Conference Proceedings 1243.

[2] _ : The centrally extended Heisenberg algebra and its connection with the Schrödinger, Galilei and Renormalized Higher Powers of Quantum White Noise Lie algebras, with Luigi Accardi, Proceedings of the VIII International Workshop Lie Theory and its Applications in Physics, Varna, Bulgaria, June 16-21, 2009, pages 115-125, American Institute of Physics, AIP Conference Proceedings 1243.

[3] _ : Renormalized higher powers of white noise (RHPWN) and conformal field theory, Infinite Dimensional Analysis, Quantum Probability, and Related Topics 9, No. 3, (2006) 353-360.

[4] _ : The emergence of the Virasoro and $w_{\infty}$ Lie algebras through the renormalized higher powers of quantum white noise, International Journal of Mathematics and Computer Science, 1, No.3, (2006) 315-342.

[5] $\quad$ : Fock representation of the renormalized higher powers of white noise and the Virasoro-Zamolodchikov- $w_{\infty} *-$ Lie algebra, J. Phys. A: Math. Theor., 41 (2008).

[6] _ Cohomology of the Virasoro-Zamolodchikov and Renormalized Higher Powers of White Noise $*-$ Lie algebras, Infinite Dimensional Anal. Quantum Probab. Related Topics, Vol. 12, No. 2 (2009) 120.

[7] __ : Quantum probability, renormalization and infinite dimensional *-Lie algebras, SIGMA (Symmetry, Integrability and Geometry: Methods and Applications), 5 (2009), 056, 31 pages.

[8] _ C C C _ Central extensions and stochastic processes associated with the Lie algebra of the renormalized higher powers of white noise, Proceedings of the 11th workshop: non-commutative harmonic analysis with applications to probability, Bedlewo, Poland, August 2008, Banach Center Publ. 89 (2010), 13-43.

[9] _ : On the central extensions of the Heisenberg algebra, QP-PQ: Quantum Probability and White Noise Analysis - vol. 25, Quantum Probability and Infinite Dimensional Analysis. Proceedings of the 29th conference, Hammamet,Tunisia,13-18, October 2008, pages 1-12.

[10] Accardi, L., Boukas, A., Misiewicz, J. : Renormalization and central extensions of Lie algebras, submitted.

[11] Accardi L., Lu Y. G., Volovich I. V.: White noise approach to classical and quantum stochastic calculi, Lecture Notes of the Volterra International School of the same title, Trento, Italy (1999), Volterra Center preprint 375, Università di Roma Tor Vergata.

[12] Bourbaki, N.: Groupes et Algebres de Lie, 2nd ed., Hermann, Paris, 1971.

[13] Feinsilver, P. J., Schott, R.: Algebraic structures and operator calculus. Volumes I and III, Kluwer, 1993.

[14] _ Differential relations and recurrence formulas for representations of Lie groups, Stud. Appl. Math., 96 (1996), no. 4, 387-406.

[15] Feinsilver, P. J., Kocik, J., Schott, R.: Representations of the Schroedinger algebra and Appell systems, Fortschr. Phys. 52 (2004), no. 4, 343-359.

[16] Franz U.: Representations et processus stochastiques sur les groupes de Lie et sur les groupes quantiques, Memoire de D.E.A., Université Henri Poincaré - Nancy I (1994)

[17] Guichardet A., Symmetric Hilbert spaces and related topics, Lect. Notes Math. 261, Springer, Berlin, 1972.

[18] Ovando, G.: Four dimensional symplectic Lie algebras, Beitrage Algebra Geom. 47 (2006), no. 2, $419-434$.

[19] Parthasarathy K.R., Schmidt K., Positive definite kernels continuous tensor products and central limit theorems of probability theory, Springer Lecture Notes in Mathematics no. 272, 1972.

Centro Vito Volterra, Universitá di Roma Tor Vergata, via Columbia 2, 00133 Roma, Italy

E-mail address: volterra@volterra.mat.uniroma2.it

$U R L:$ http://volterra.mat.uniroma2.it

Department of Mathematics, American College of Greece, Aghia Paraskevi 15342, Athens, Greece

E-mail address: andreasboukas@acg.edu

University of Zielona Góra, Department of Mathematics, Poland

E-mail address: J.Misiewicz@wmie.uz.zgora.pl 